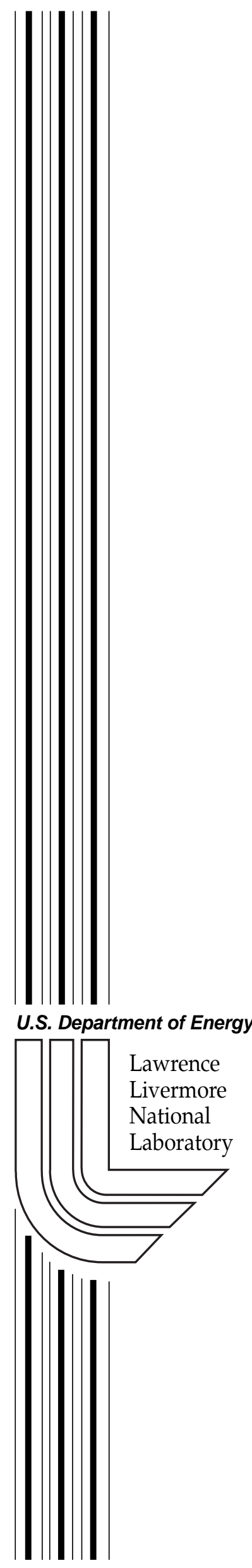

Preprint

UCRL-ID-152901

\title{
16-APR-03 Final Release of ENDF/B-V for use with LLNL Codes
}

T. S. Hill, D. P. McNabb, G. W. Hedstrom, B. Beck, C. A. Hagmann

\section{April 16, 2003}




\section{DISCLAIMER}

This document was prepared as an account of work sponsored by an agency of the United States Government. Neither the United States Government nor the University of California nor any of their employees, makes any warranty, express or implied, or assumes any legal liability or responsibility for the accuracy, completeness, or usefulness of any information, apparatus, product, or process disclosed, or represents that its use would not infringe privately owned rights. Reference herein to any specific commercial product, process, or service by trade name, trademark, manufacturer, or otherwise, does not necessarily constitute or imply its endorsement, recommendation, or favoring by the United States Government or the University of California. The views and opinions of authors expressed herein do not necessarily state or reflect those of the United States Government or the University of California, and shall not be used for advertising or product endorsement purposes.

This is a preprint of a paper intended for publication in a journal or proceedings. Since changes may be made before publication, this preprint is made available with the understanding that it will not be cited or reproduced without the permission of the author.

This work was performed under the auspices of the United States Department of Energy by the University of California, Lawrence Livermore National Laboratory under contract No. W-7405-Eng- 48 .

This report has been reproduced directly from the best available copy.

Available electronically at http://www.doc.gov/bridge

Available for a processing fee to U.S. Department of Energy

And its contractors in paper from

U.S. Department of Energy

Office of Scientific and Technical Information

P.O. Box 62

Oak Ridge, TN 37831-0062

Telephone: (865) 576-8401

Facsimile: (865) 576-5728

E-mail: reports@adonis.osti.gov

Available for the sale to the public from

U.S. Department of Commerce

National Technical Information Service

5285 Port Royal Road

Springfield, VA 22161

Telephone: (800) 553-6847

Facsimile: (703) 605-6900

E-mail: orders@ntis.fedworld.gov

Online ordering: http://www.ntis.gov/ordering.htm

\section{OR}

Lawrence Livermore National Laboratory

Technical Information Department's Digital Library

http://www.llnl.gov/tid/Library.html 
The Computational Nuclear Physics Group in N Division is issuing the first official release of the ENDF/B-V evaluations for users of ndf, mcf and TART data files. The new files can be found on OCF (blue and compass) and SCF (sky and forest) in the appropriate subdirectories of

\section{/usr/gapps/data/nuclear/endfb5/endfb5r2 and /usr/gapps/data/nuclear/endfb5/endfb5r2+end199.}

These data files are in Cray binary format. The $\mathbf{p d b}$ versions of the mef data have also been prepared for CMCAPM users (fortran version unsupported).

The new data files were prepared in two steps. First, the ENDF/B-V database was translated to an ENDL-format ascii database. The ENDL ascii format is a point-wise tabular storage scheme where intermediate values are extracted via interpolation. Sufficient point-wise information was generated in the translation to insure an extraction tolerance of $0.1 \%$ for most of the data. The only exception is along the incident neutron energy axis of the outgoing particle energy probability density function where a $0.5 \%$ tolerance was maintained. Second, processed files were generated from the translated database. Since the translated ENDF/B-V data is in ENDLformat, the standard processing codes were used to generate the new processed data files. To the best of our knowledge, these processed data files are accurate representations of the ENDF/B-V database to within the stated tolerances. However, there are several issues that users must be aware of:

1. The ENDF/B-V database does not contain a carbon 12 evaluation. After the translation, a copy of the natural carbon evaluation (za006000) was added and renamed carbon 12 (za006012). The carbon 12 file was added to enhance compatibility since the ENDL database does not contain natural carbon. Technically, this is acceptable since natural carbon is $98.9 \%$ carbon 12 .

2. The format of the ENDF/B-V database severely limits the amount of information that can be stored about outgoing charged particles. Cross sections with exit channels containing charged particles are numerous; however, outgoing charged particle energy and angular distributions are not. As part of the processing, we determine the "missing" charged particle energy from kinematics and deposit it locally.

3. The ENDF/B-V gamma-ray production information is often not associated with particular exit channels. Instead, the (n,Xgamma) channel is used to store the gamma-rays which are not explicitly associated with particular reactions. Summing the gamma-ray production channels in order to determine a single gamma-ray source term for each isotope will produce the desired result.

4. The format of the ENDF/B-V database provides no means of storing double differential data. Therefore, the angular and energy information of outgoing particles is completely uncorrelated. 
5. There are 110 targets in ENDL94/ENDL99 and 124 in ENDF/B-V; only 68 of them are common to both databases. The lists of targets are presented below in order of atomic number $\left(\mathrm{Z}^{*} 1000\right)$ plus mass number $(\mathrm{A})$. The common targets are in bold print.

ENDL94 Targets

za000001 za001001 za001002 za001003 za002003 za002004 za003006 za003007 za004007 za004009 za005010 za005011 za006012 za006013 za007014 za007015 za008016 za009019 za010020 za011023 za012000 za013027 za014000 za015031 za016032 za017000 za018000 za019000 za020000 za022000 za023051 za024000 za025055 za026000 za027059 za028000 za028058 za029000 za030000 za031000 za033074 za033075 za039088 za039089 za040000 za041093 za042000 za047107 za047109 za048000 za049000 za050000 za051000 za053127 za054000 za054134 za056138 za063000 za064000 za067165 za072000 za073181 za074000 za075185 za075187 za078000 za079197 za080000 za082000 za083209 za090231 za090232 za090233 za091233 za092233 za092234 za092235 za092236 za092237 za092238 za092239 za092240 za093235 za093236 za093237 za093238 za094237 za094238 za094239 za094240 za094241 za094242 za094243 za095241 za095242m za095243 za096242 za096243 za096244 za096245 za096246 za096247 za096248 za097249 za098249 za098250 za098251 za098252 za099120 za099125

\section{ENDF/B-V Targets}

za001001 za001002 za001003 za002003 za002004 za003006 za003007 za004009 za005010 za005011 za006000 za007014 za007015 za008016 za008017 za009019 za011023 za012000 za013027 za014000 za015031 za016000 za016032 za017000 za019000 za020000 za022000 za023000 za024000 za025055 za026000 za027059 za028000 za029000 za031000 za036078 za036080 za036082 za036083 za036084 za036086 za037085 za037087 za040000 za040090 za040091 za040092 za040094 za040096 za041093 za042000 za043099 za045103 za047107 za047109 za048000 za048113 za054124 za054126 za054128 za054129 za054130 za054131 za054132 za054134 za054135 za054136 za055133 za056138 za062149 za063000 za063151 za063152 za063153 za063154 za064152 za064154 za064155 za064156 za064157 za064158 za064160 za066164 za071175 za071176 za072000 za072174 za072176 za072177 za072178 za072179 za072180 za073181 za073182 za074000 za074182 za074183 za074184 za074186 za075185 za075187 za079197 za082000 za083209 za090232 za091233 za092233 za092234 za092235 za092236 za092238 za093237 za094238 za094239 za094240 za094241 za094242 za095241 za095242m za095243 za096243 za096244 za096245 za096246 za099121 za099122

6. In addition to the target inventory of ENDF/B-V, we have included the two fission fragment files generated and used at Los Alamos. These fission fragment files, listed as za099121 and za099122, are "typical" fission fragments for uranium (za045117) and plutonium (za046119). These files are an average over evaluations performed for the ten most common fission fragments for each actinide. 
7. Many of the ENDF/B-V evaluations have no gamma-ray information. For these 66 evaluations we add an ( $\mathrm{X}, \mathrm{Xgamma}$ ) cross section which is zero over the entire incident neutron energy range. The presence of these null files insures that these isotopes are listed in the photon production libraries. As a result, users can choose to track photons without modifying their assemblies or codes. Below is the list of evaluations which have no gammaray information:

za001003 za002003 za002004 za005011 za008017 za036078 za036080 za036082 za036083 za036084 za036086 za037085 za037087 za040000 za040090 za040091 za040092 za040094 za040096 za043099 za045103 za047107 za047109 za048000 za048113 za054124 za054126 za054128 za054129 za054130 za054131 za054132 za054134 za054135 za054136 za055133 za062149 za063000 za063152 za063154 za064152 za064154 za064155 za064156 za064157 za064158 za064160 za066164 za071175 za071176 za072000 za072174 za072176 za072177 za072178 za072179 za072180 za073182 za075185 za075187 za079197 za091233 za092234 za092236 za093237 za094238

8. The ENDF/B-V database contains only neutron-induced reactions and therefore only those data files were processed. Below is a listing of the new files residing in the endfb5r2 directory:

\begin{tabular}{clc} 
Directory structure & Description \\
\hline mcf/ & & mcf data sub-directory \\
& mcf1 & neutron data \\
ndf/ & mcf1.pdb & neutron data (pdb format) \\
& ndf1.087 & ndf data sub-directory \\
& ndf1.175 & 87 group neutron data (bdfls id=4) \\
& ndf1.230 & 175 group neutron data (bdfls id=6) \\
tart/ & & 230 group neutron data (bdfls id=7) \\
& tartppd & TART data sub-directory \\
& tartnd & 175 group photon production data \\
& cross & 175 group neutron interaction data \\
& & type-2 banding data
\end{tabular}

\begin{tabular}{|c|c|c|c|c|}
\hline File & Date & sha output (i.e., checksum) & & \\
\hline $\mathrm{mcf} / \mathrm{mcf} 1$ & 030301 & $\mathrm{a} 0647968 \quad 359 \mathrm{eb} 35 \mathrm{e}$ b253b4d3 & ealaadef & $\mathrm{c} 877990 \mathrm{a}$ \\
\hline mcf/mcf1.pdb & 030302 & le6606a4 a32b7b51 536d86ed & $9 f d f c e 3 c$ & $d 9 e 3723 d$ \\
\hline ndf/ndf1.087 & 030303 & $4 a 7 b f 261$ aebdaalb dd93d254 & $25 e a 49 a 3$ & $91003 d 30$ \\
\hline ndf/ndf1.175 & 030304 & $30960 a 2 b$ cf $7 c 5 a 219 b 6 e 15 c 4$ & $4 \mathrm{~d} 8 \mathrm{cb} 070$ & $5 \mathrm{a} 6732 \mathrm{ce}$ \\
\hline $\mathrm{ndf} / \mathrm{ndf1.230}$ & 030305 & e5d69525 51199d62 c4fcc0a2 & b37af053 & $9 \mathrm{~d} 8 \mathrm{~b} 6122$ \\
\hline tart/tartppd & 030309 & $198 f f 812 f 873784 d \quad 0 \mathrm{cc} 60 \mathrm{c} 60$ & 2928 ad6d & $\mathrm{b} 20 \mathrm{ffccc}$ \\
\hline tart/tartnd & 030308 & $169 a b 7 c 8$ bleb3171 fa6c74e9 & eb7d9d59 & $335 \mathrm{e} 2 \mathrm{c} 64$ \\
\hline tart/cross & 030306 & $82 \mathrm{~b} 4 \mathrm{ae} 70$ e6204cdc $156 \mathrm{~b} 48 \mathrm{ab}$ & $7 \mathrm{bc} 9 \mathrm{cc} 92$ & $\mathrm{fb} 94428 \mathrm{a}$ \\
\hline
\end{tabular}


9. We anticipate that some users and code developers may wish/need to avoid modifying assembly compositions due to missing evaluations in the ENDF/B-V data. For these users we have constructed a hybrid library which contains the ENDF/B-V evaluations and the unique ENDL evaluations. This hybrid library allows user codes to run without modification to their assembly and gain access to the ENDF/B-V data when it is available. Below is a listing of the new files residing in the endfb5r2+endl99 directory:

\begin{tabular}{|c|c|c|}
\hline \multicolumn{2}{|c|}{ Directory structure } & Description \\
\hline mcfl & & mcf data sub-directory \\
\hline & mcf1 & neutron data \\
\hline & mcf1.pdb & neutron data ( $p d b$ format) \\
\hline ndf/ & & ndf data sub-directory \\
\hline & ndf1.087 & 87 group neutron data (bdfls id $=4)$ \\
\hline & ndf1.175 & 175 group neutron data (bdfls id $=6$ ) \\
\hline & ndf1.230 & 230 group neutron data (bdfls id $=7$ ) \\
\hline tart/ & & TART data sub-directory \\
\hline & $\begin{array}{l}\text { tartppd } \\
\text { tartnd } \\
\text { cross }\end{array}$ & $\begin{array}{l}175 \text { group photon production data } \\
175 \text { group neutron production data } \\
\text { type-2 banding data. }\end{array}$ \\
\hline
\end{tabular}

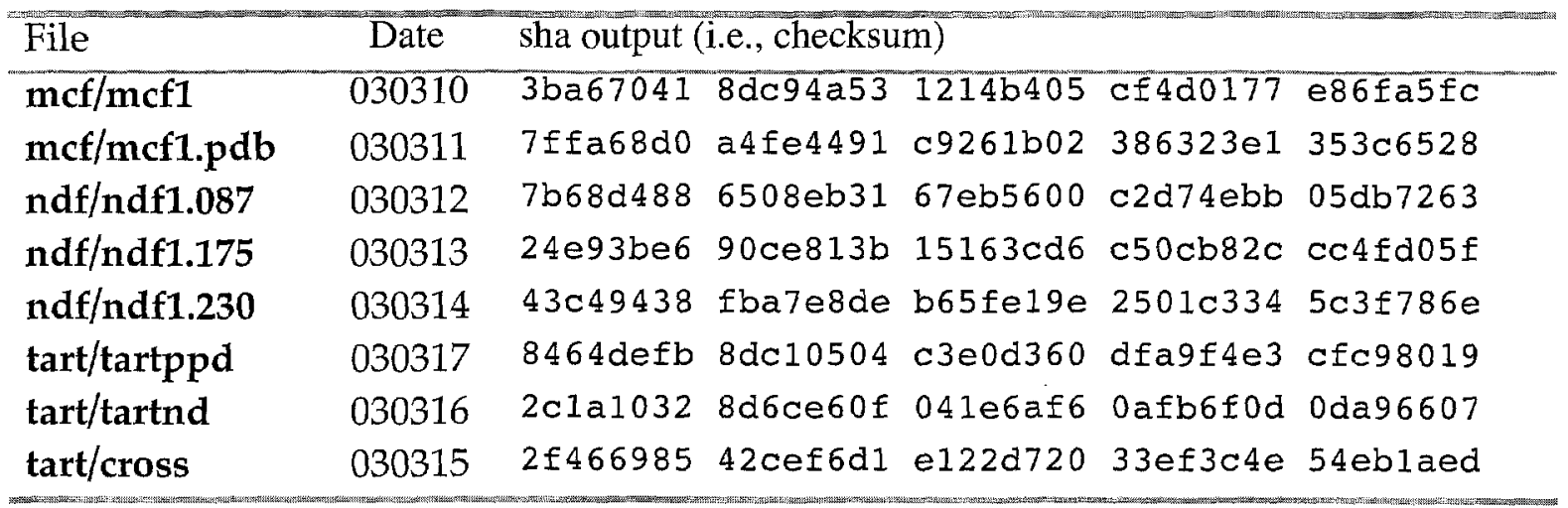

Testing has been carried out using a variety of LLNL codes to insure basic compatibility. However, given the limited information present in the ENDF/B-V database, some client codes may not run in the "standard" mode and users need to carefully consider the issues listed above. 